\title{
A Case Of Carcinoid Testis
}

\author{
Dr.Rajeev Thilak C ${ }^{1}$, Dr.Thirunavukarasu², Dr.Karthikeyan ${ }^{3}$, Dr.Ameesh Izath ${ }^{4}$. \\ (Institue Of General Surgery, Madras Medical College, Chennai,India)
}

\begin{abstract}
Testicular cancers account for around 1-1.5\% of male neoplasms. A vast majority are germ cell tumours (more than 90\%) followed by lymphomas (around 5\%) and interstitial tumours (around 2\%). Carcinoid tumour of the testis is a rare tumour accounting for less than 1\% of all testicular tumours. It is usually confined to the testis. Fewer than 100 cases have been reported in the literature.
\end{abstract}

Keywords: Carcinoid, Testis

\section{Introduction}

Testicular carcinoid tumours are very rare and these tumours are mainly classified into three distinct groups, most commonly primary testicular carcinoid, carcinoid differentiation within a mature teratoma,and metastases from an extra-testicular source. Testicular carcinoid tumours can present in any age group starting from age of ten upto age of eighty years ${ }^{2}$. Most of the testicular carcinoid tumours may be with self-detected testicular mass or testicular ache as with common testicular tumours, or uncommonly with carcinoid syndrome.We report a case of primary carcinoid tumour of the testis without features of carcinoid syndrome.

\section{Case Report}

27 year old male was admitted with complaints of pain abdomen for 8 months, Mass abdomen for 6 months, Scrotal swelling for 4 months. The abdominal pain was intermittent, dull aching in nature and progressively increasing in severity. The abdominal mass was located on the left side which was initially small and gradually increased in size. He noticed a scrotal swelling for 4 months which gradually increased in size. It was not associated with pain or trauma. He had history of loss of appetite and loss of weight. There was no history of fever / chest pain / breathlessness / cough / vomiting / hematemesis / malena / altered bowel habits. On examination, he was thin built and moderately nourished. He was not anemic/ icteric. He had left axillary and Bilateral Inguinal lymph nodes which were soft and $<1 \mathrm{~cm}$ in size. Abdominal examination revealed a mass of size approximately $15 * 10 \mathrm{~cm}$ in size palpable involving Left hypochondrium, Left iliac fossa, umbilical and Left lumbar regions which was hard in consistency, had nodular surface, was immobile and not bimanually palpable or ballotable. All borders were well made out. Upper border was $2 \mathrm{~cm}$ below costal margin at Left midclavicular line, lower border at the level of anterior superior iliac spine, medially extended about $2 \mathrm{~cm}$ to the right of midline and laterally $3 \mathrm{~cm}$ to the left of Left mid-clavicular line. There was no hepatomegaly or free fluid. Supraclavicular fossa was free. Examination of the scrotum showed $6 * 4 \mathrm{~cm}$ enlarged left testis, which had a smooth surface and was hard in consistency. There was loss of testicular pain sensation on the left side. The provisional diagnosis was left testicular tumour with retroperitoneal lymphadenopathy probably due to seminoma. Routine blood investigations namely CBC, RFT and LFT were normal. Tumour markers were done and LDH and $\beta H C G$ levels were elevated [LDH - $1454 \mathrm{U} / \mathrm{L} ;$ AFP $-1.14 \mathrm{IU} / \mathrm{ml} ; \beta \mathrm{HCG}-71.6 \mathrm{mIU} / \mathrm{ml}]$. Ultrasound scrotum showed an enlarged left testis with multiple loculations with altered echogenicity with mass lesion near lower pole. Ultrasound abdomen showed conglomerate retroperitoneal para-aortic nodal mass $14 * 7$ $\mathrm{cm}$ in size.

CT abdomen showed a $15 * 20 \mathrm{~cm}$ well defined heterodense lobulated lesion with specks of calcification involving left para-aortic region from L2 to L4 encasing Aorta, IVC, Left Renal vein, coeliac plexus, Superior mesenteric Vein ; compressing upper pole of Left kidney \& Pelvi-ureteric junction causing Lt hydronephrosis; infiltrating Lt psoas muscle; small intestine was lifted anteriorly. There was no hepatomegaly and no intestinal abnormalities were noted. The final impression was retroperitoneal necrotic lymph node. 

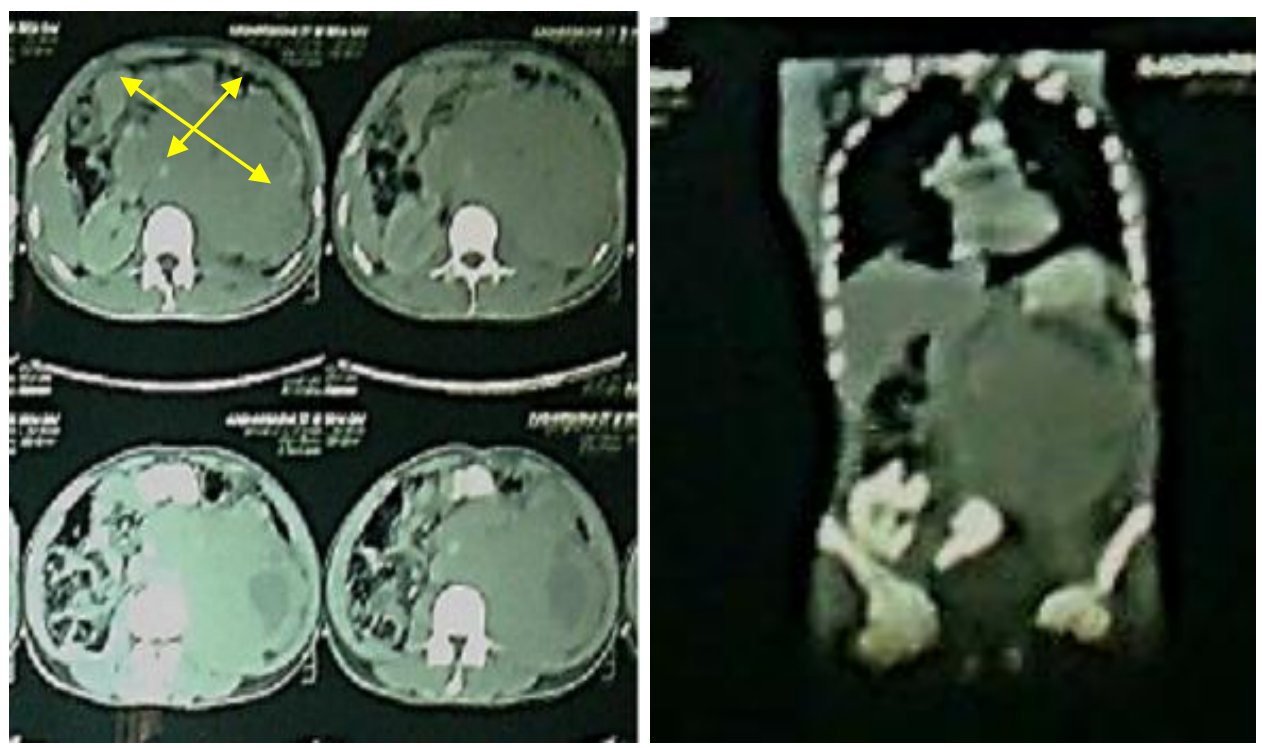

CT chest was found to be normal. FNAC of axillary and inguinal lymph nodes showed only inflammatory changes.

Left high inguinal orchidectomy was done and a $2 * 2 \mathrm{~cm}$ tumour occupying lower pole of the testis was found. Cut surface had a Cut potato like appearance and was sent for histopathological analysis.
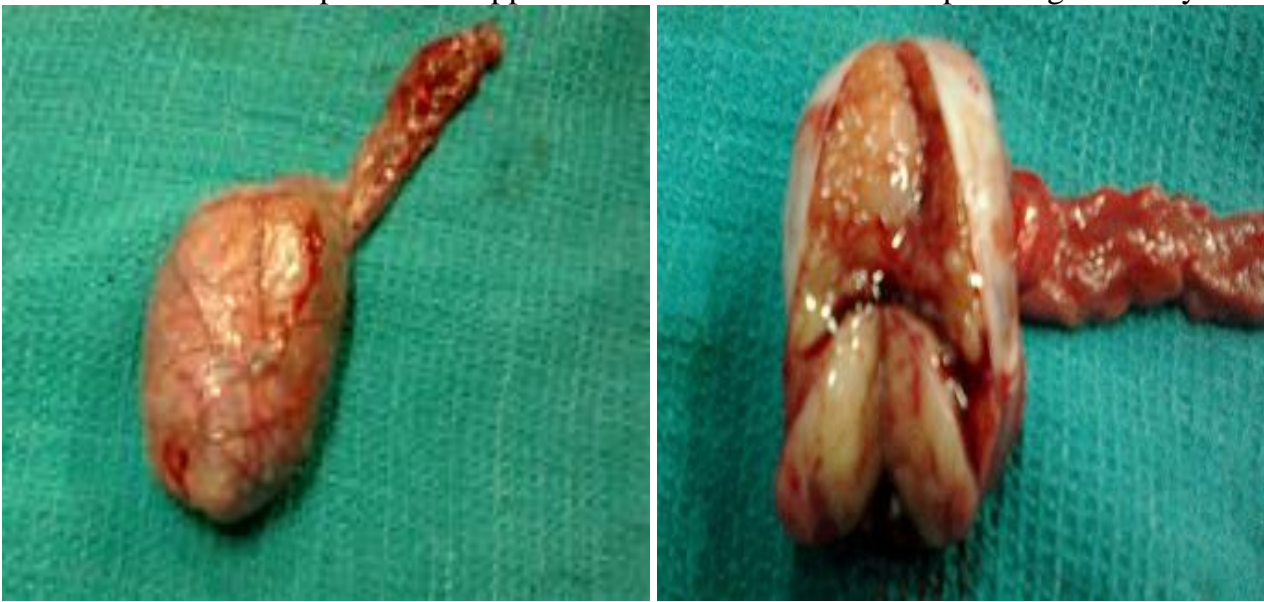

HPE report was given as external surface - smooth and cut surface showing multiple grey white nodules largest $2 \mathrm{~cm}$ interspersed amidst normal parenchyma. Nodule is grey white with focal yellowish areas showing nests of round to oval cells separated by vascular fibrous stroma; tumour cells are uniform in appearance with coarse chromatin pattern; adjacent Blood vessels show tumour emboli; tubule shows atrophy; no infiltration into epididymis/sac wall; resected end of the cord - normal. The final impression was neuroendocrine well differentiated (carcinoid) tumour of the testis. Immunohistochemistry analysis showed weak positivity for Synaptophysin \& Chromogranin and diffuse strong positivity for Neuron specific enolase.

The case was discussed in tumour board and was categorised as stage IIIA $\left(\mathrm{T}_{1} \mathrm{~N}_{3} \mathrm{M}_{0} \mathrm{~S}_{2}\right)$. He was put on BEP (Bleomycin, Etoposide, Cisplatin) chemotherapy for three cycles and showed more than $50 \%$ reduction in size of nodal mass.

\section{Discussion}

Carcinoid tumours of the testis constitute less than one percent of testicular tumours. ${ }^{1}$ The first reported case of primary testicular carcinoid was described by Simon et al. in 1954. ${ }^{2}$ A painless mass and notable testicular enlargement are the two most common presenting features. It usually affects older males (40-60 years). They can be encountered in 3 clinical settings: as a de novo neuroendocrine neoplasm (most common); as a component of a teratomatous tumour; as a metastatic lesion from extra-testicular primary tumour (least common). ${ }^{2}$ The cell of origin is debatable and may arise from the same progenitor cells from which Leydig cells arise $^{4}$ or may have a germ cell origin. ${ }^{3}$

The majority are only diagnosed on histopathology as they do not become clinically apparent until there is metastatic spread or the presence of carcinoid syndrome. ${ }^{2}$ Carcinoid tumours of testis rarely metastasize, 
with the overall incidence estimated at $11 \%$. Features of carcinoid syndrome are seen in $<10 \%$ of cases $^{5}$ and typically occur only once the tumour has metastasised to the liver or lungs. Elevated serotonin levels and that of its metabolite 5-hydroxyindoleacetic acid (5-HIAA) are present in the blood and urine of those patients with this syndrome.

It is necessary to exclude the presence of a primary tumour in another organ before confirming the diagnosis of a primary testicular carcinoid tumour as around $10 \%$ of cases are due to metastasis from another location. ${ }^{2}$ Investigations to locate primary include CT abdomen, Barium Contrast studies, $\mathrm{x}$-ray/CT Chest , $\mathrm{x}$ ray/CT Pelvis, Somatostatin receptor scintigraphy using indium-111 labelled octreotide. ${ }^{7}$ The latter is superior to CT in localization of primary tumour site and has a $96 \%$ sensitivity for detecting metastases. ${ }^{6}$

Pathologically, the lesions are described as solid yellow-tan in appearance with an exceedingly firm texture due to striking desmoplasia which is characteristically present. Histologically, the neoplastic cells can form discrete islands, trabeculae, strands, glands, or undifferentiated sheets. Immunohistochemical studies show reactivity to antibodies to cytokeratin AE1 and AE3, Chromogranin-A, neuron specific enolase, Synaptophysin, and CD56. ${ }^{8}$

High orchidectomy remains the main modality of treatment and is curative if tumour is confined to the testis. ${ }^{5}$ Chemotherapy is not usually indicated unless there is metastasis. The long-term prognosis of carcinoid tumours is dependent on size, association with teratoma and presence of metastases. Larger tumour and the presence of carcinoid syndrome predicted increased metastatic potential and hence poorer prognosis. The prognosis of carcinoid tumours arising within teratoma is better than pure testicular carcinoid. ${ }^{9}$ Long term biochemical and radiological follow-up is essential given potential for delayed metastases. ${ }^{10}$ Urine 5-HIAA (every 3 months for 1 year and then yearly thereafter) ${ }^{11}$ and serum Chromogranin-A levels are used for followup.

\section{Conclusion}

Carcinoid tumour of the testis is a rare tumour accounting for less than $1 \%$ of all testicular tumours. It can occur de novo or it can be present as a component of a teratoma. Metastasis from carcinoid tumours at other sites also has to be excluded by appropriate investigations. It usually presents as a painless testicular enlargement. Lymph node metastasis is exceedingly rare. Distant metastasis and thus carcinoid syndrome can occur in $10 \%$ of cases. High orchidectomy is the mainstay of treatment and Chemotherapy is not indicated unless there is metastasis. Prognosis depends on tumour size and presence or absence of carcinoid syndrome. Long term biochemical and radiological follow-up is essential due to the potential for delayed metastases.

\section{Acknowledgement}

I would like to thank Dr. Thirunavukarasu M.S for his immense support and guidance.

I would like to thank my institute, IGS, Madras Medical College for their support.

Finally, my colleagues Dr.Karthikeyan and Dr.Ameesh Izath for their valuable assistance.

[1]. T. Hayashi, S. Iida, J. Taguchi et al., "Primary carcinoid of the testis associated with carcinoid syndrome," International Journal of Urology, vol. 8, no. 9, pp. 522-524, 2001.

[2]. Stroosma OB, Delaere KPJ. Carcinoid tumours of the testis. Br J Urol. 2008; 101(9): 1101-5.

[3]. Abbosh PH, Zhang S, Maclennan GT, et al: Germ cell origin of testicular carcinoid tumors. Clin Cancer Res 14:1393-1396, 2008

[4]. Mai KT, Park PC, Yazdi HM, Carlier M. Leydig cell origin of testicular carcinoid tumour: immunohistochemical and electron microscopic evidence. Histopathology. 2006; 49(5): 548-58.

[5]. Wolf M, Wunderlich H, Hindermann W, Gajda M, Schreiber G, Schubert J. Case report: Primary carcinoid tumour of the testicle without metastases in combination with testicular atrophy and testosterone deficiency. Int Urol Nephrol. 2006; 38(3-4):625-8.

[6]. D. Neely and S. Gray, "Primary carcinoid tumour of the testis," Ulster Medical Journal, vol.80 (2), pp. 79-81, 2011.

[7]. Ramage JK, Ramage JK, Davies AH, Ardill J, Bax N, Caplin M, Grossman A, et al. Guidelines for the management of gastroenteropancreatic neuroendocrine (including carcinoid) tumours. Gut. 2005; 54(suppl 4): iv1-16.

[8]. V. Kumar, R. S. Cotran, and S. L. Robbins, Robbins Basic Pathology, Saunders, Philadelphia, Pa, USA, 2003.

[9]. A. Zavala-Pompa, J. Y. Ro, A. El-Naggar et al., "Primary carcinoid tumor of testis: immunohistochemical, ultrastructural, and DNA flow cytometric study of three cases with a review of the literature," Cancer, vol. 72, no. 5, pp. 1726-1732, 1993. 
[10]. Hayashi T, Iida S, Taguchi J, Miyajima J, Matsuo M, Tomiyasu K, et al. Primary carcinoid of the testis associated with carcinoid syndrome. Int J Urol. 2001; 8(9):522-4.

11. Sutherland RS, Wettlaufer JN, Miller GJ. Primary carcinoid tumour of the testicle: a case report and management schema. J Urol. 1992; 148(3): 880-2. 